\title{
Guy Mollet und die Entstehung der Römischen Verträge 1956/1957
}

\author{
Wilfried Loth*
}

Erfolge haben immer viele Väter. Für die europäische Einigung freilich gilt, dass sie nur vorankam, wenn sich die Spitzen der französischen und der deutschen Regierung auf konkrete Schritte verständigten, die Kompromisse beinhalteten. Die deutsch-französische Verständigung war und ist keine hinreichende Bedingung für europapolitischen Erfolg, wohl aber eine notwendige Bedingung. Das lässt sich nicht nur anhand des Schuman-Plans zeigen, mit dem Robert Schuman und Konrad Adenauer den Prozess der europäischen Einigung 1950 in Gang setzten. Auch die Römischen Verträge, die am 25. März 1957 unterzeichnet wurden, beruhten letztlich auf einer Verständigung zwischen französischer und deutscher Politik, in diesem Fall zwischen Konrad Adenauer und dem französischen Ministerpräsidenten Guy Mollet. ${ }^{1}$

\section{Die Krise des Spaak-Komitees}

Als Guy Mollet am 31. Januar 1956 französischer Ministerpräsident wurde, befand sich das europäische Projekt nämlich in einer durchaus kritischen Phase. Die Außenminister der sechs Gründungsmitglieder der Montanunion hatten zwar auf der Konferenz von Messina beschlossen, eine Studiengruppe über die Vorschläge zur Bildung einer Wirtschaftsgemeinschaft und einer Atomgemeinschaft beraten zu lassen. Ob bei diesen Beratungen aber etwas herauskommen würde, war mehr als fraglich. Genauer gesagt: Die Verhandlungen des Studienkomitees standen kurz vor dem Scheitern. Der belgische Außenminister Paul-Henri Spaak, dem der Vorsitz in diesem Komitee übertragen worden war, wusste sich nicht anders zu helfen, als die Verhandlungen Ende November 1955 erst einmal zu unterbrechen.

In Frankreich gab es erhebliche Bedenken, die Wirtschaft der ungehinderten Konkurrenz eines Gemeinsamen Marktes auszusetzen. Sie hatte sich noch kaum von den Folgen der Weltwirtschaftskrise und der deutschen Besatzung erholt und war in mancher Hinsicht noch nicht konkurrenzfähig. Gleichzeitig waren im Zuge der Modernisierung Sozialleistungen erkämpft worden, die diejenigen, die davon profitierten, natürlich nicht der Rückkehr zu liberalen Prinzipien opfern wollten. Folglich sperrten sich die französischen Vertreter im sogenannten SpaakKomitee gegen die Forderung der Deutschen und der Niederländer nach einem verbindlichen Zeitplan zum vollständigen Abbau der Handelshemmnisse und der Entwicklung einer gemeinsamen Zoll- und Handelspolitik. Stattdessen beharrten sie auf flexibler Reaktion auf die konjunkturelle Entwicklung, Harmonisierung der Sozialkosten und einem gemeinsamen Investitionsfonds, der das Aufholen rückständiger Branchen und Regionen erleichterte - Vorstellungen, die die deutschen Marktwirtschaftler als Sünden wider die ökonomische Vernunft betrachteten. ${ }^{2}$

Prof. Dr. Wilfried Loth, Historisches Institut, Abteilung für Neuere und Neueste Geschichte, Universität Duisburg/Essen.

1 Ausführlicher wird diese Thematik behandelt in: Wilfried Loth: Deutsche und französische Interessen auf dem Weg zu EWG und Euratom, in: Andreas Wilkens (Hrsg.): Deutsch-französische Wirtschaftsbeziehungen 1945-1960, Sigmaringen 1997, S. 171-187.

2 Vgl. die Verhandlungsberichte bei Hanns Jürgen Küsters: Die Gründung der europäischen Wirtschaftsgemeinschaft, Baden-Baden 1982, S. 135-218 und 232-251, und Michel Dumoulin: Les travaux du Comité Spaak (juillet 1955 - avril 1956), in: Enrico Serra (Hrsg.): Il Rilancio dell'Europa e i Trattati di Roma, Milano 1989, S. 195-210. Dazu die Zeugnisse von Hans von der Groeben und Baron Snoy, ebenda, S. 294-300, sowie die Materialien zum EWG-Vertrag im Politischen Archiv des Auswärtigen Amtes (PAAA), Büro Staatssekretär (Büro StS), Bd. 350. 
Umgekehrt zeigten die deutschen Vertreter wenig Neigung, der vom französischen Delegationsleiter Félix Gaillard mit Nachdruck geforderten Atomgemeinschaft näher zu treten. Ursprünglich war das eine Idee, die Louis Armand, damals Präsident der nationalen Eisenbahngesellschaft, im Kontakt mit Jean Monnet entwickelt hatte. Es ging ihnen dabei um die Zurüstung Frankreichs und der übrigen Europäer für die dritte industrielle Revolution, wobei sie in damals noch ungebrochener technischer Fortschrittseuphorie glaubten, dass ein solches Projekt besonders viel Zustimmung für die europäische Sache mobilisieren müsste. Die nationale Atomenergie-Kommission stand dem Vorhaben zwar skeptisch gegenüber; aber nachdem geklärt war, dass die Atomgemeinschaft nicht auf den militärischen Bereich ausgedehnt werden sollte, gab es eine parlamentarische Mehrheit, die es positiv sah. Dies war für die Regierung Edgar Faure (die zuvor amtierende französische Regierung) Grund genug, das Vorhaben in den Vordergrund der Verhandlungen zu stellen. ${ }^{3}$

Davon war nun die deutsche Seite überhaupt nicht erbaut. Sonderminister Franz-Josef Strauß, seit Oktober 1955 mit der Verantwortung für die Atomfrage betraut, war zu diesem Zeitpunkt viel mehr an einer Zusammenarbeit mit den Briten und Amerikanern interessiert, die in der Atomtechnik viel weiter vorangekommen waren als die Franzosen. In einer Europäischen Atomgemeinschaft sah er ein Unternehmen, das in erster Linie Frankreich zugutekommen sollte. Sich dafür herzugeben, bestand aus seiner Sicht für die Bundesrepublik keine Veranlassung. Eine Annäherung der gegensätzlichen Standpunkte war nicht zu erzielen; da passte es ganz gut, dass am 29. November in Paris die Regierung Faure ihre parlamentarische Mehrheit verlor. Spaak nützte die Regierungskrise in Frankreich, um die Verhandlungen bis auf Weiteres zu vertagen. ${ }^{4}$

Dass in dieser Situation Mollet neuer Ministerpräsident in Frankreich wurde, hatte durchaus etwas mit der Krise der Verhandlungen über Wirtschaftsgemeinschaft und Atomgemeinschaft zu tun. Nach den Neuwahlen vom 2. Januar 1956 war eine arbeitsfähige Mehrheit in der französischen Nationalversammlung nur noch zu finden, wenn die christdemokratischen Volksrepublikaner und die Sozialisten zusammenwirkten. Die Christdemokraten aber, die mit Schuman zu Beginn der 1950er Jahre den Initiator der neuen Europapolitik gestellt hatten, wollten nicht wieder Pierre Mendès France als Ministerpräsidenten, den designierten Kandidaten des Parteienbündnisses aus Linksliberalen und Sozialisten, der sogenannten ,Republikanischen Front‘. Mendès France, der beim Scheitern der Europäischen Verteidigungsgemeinschaft (EVG) 1954 Ministerpräsident gewesen war, galt den Christdemokraten seither als ein Europa-Skeptiker; den Generalsekretär der Sozialistischen Partei schätzten sie dagegen als einen zuverlässigen Europa-Förderer. ${ }^{5}$

Man sieht also, dass Staatspräsident René Coty sehr genau wusste, was er tat, als er Mollet mit der Regierungsbildung beauftragte und nicht, wie es nach den Absprachen zwischen Sozialisten und Liberalen zunächst zu erwarten gewesen wäre, Mendès France. ${ }^{6}$ Die gleiche europäische Perspektive galt für die Wahl des Außenministers: Christian Pineau von der So-

3 Vgl. Pierre Guillen: La France et la négociation du traite d'Euratom, in: Relations internationales 44/1985, S. 391-412; Pierre Guillen: La France et la négociation du traité d'Euratom, in: Michel Dumoulin/Pierre Guillen/ Maurice Vaisse (Hrsg.): L'Énergie nucléaire en Europe. Des origines à Euratom, Bern usw. 1993, S. 111-129.

4 Vgl. Paul-Henri Spaak: Memoiren eines Europäers, Hamburg 1969, S. 309-314.

5 Zur innenpolitischen Situation Frankreichs in der Entstehungsphase der Römischen Verträgen vgl. etwa Serge Berstein/Pierre Milza: Histoire de la France au XXe siècle. Tome III: 1945-1958, Bruxelles 1991, S. 266-271.

6 Zur Person von Guy Mollet vgl. die Biographie von François Lafon: Guy Mollet. Itinéraire d'un socialiste controversé (1905-1975), Paris 2006; zu seiner Europapolitik auch Wilfried Loth: Sozialismus und Internationalismus. Die französischen Sozialisten und die Nachkriegsordnung Europas 1940-1950, Stuttgart 1977; Wilfried Loth: The French Socialist Party, 1947-1954, in: Richard Th. Griffith (Hrsg.): Socialist Parties and the Question of Europe in the 1950's, Leiden/New York 1993, S. 25-41. 
zialistischen Partei. Sie galt auch für die Bestellung eines Staatssekretärs für Europafragen, ein Amt, das der Generalsekretär der Radikalen Partei Maurice Faure übernahm. ${ }^{7}$ Und sie galt auch für die Wahl des Kabinettschefs von Mollet: Der neue Regierungschef berief seinen engsten europapolitischen Mitarbeiter in dieses Amt, den Sozialisten Emile Noël. Mollet wusste, dass die Überwindung der europapolitischen Krise die wichtigste Aufgabe war, vor der seine Regierung stand; und er tat sein Bestes, um sich für diese Aufgabe zu rüsten. Als Pineau später während der Suez-Krise an Rücktritt dachte, beschied ihm Mollet: „,Vergessen Sie nicht, dass Sie am Quai d'Orsay sind, um Europa zu machen!‘8

\section{Mollets Krisenstrategie}

Für die Europäer der Republikanischen Front ging es also darum, einen Ausweg aus der Sackgasse zu finden. ,Wir hatten den Eindruck“, berichtete Pineau, ,dass wir vor einer großen Herausforderung standen. Was wir vor allem fürchteten, und das war ganz entscheidend, war ein Vertrag, der nicht ratifiziert werden würde. Denn wenn Frankreich nach dem Scheitern der EVG in diesem Moment erneut den Vorwand für ein Scheitern Europas geliefert hätte, dann hätten wir uns endgültig diskreditiert und dann hätten wir uns an der Schaf-

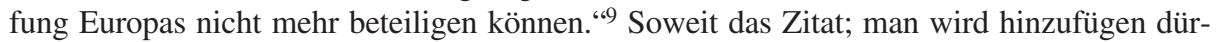
fen: dann wäre es mit der Schaffung Europas auch nicht mehr weitergegangen, jedenfalls für absehbare Zeit. Das bedeutete, dass man die Verhandlungen so führen musste, dass anders als bei der EVG zum Schluss eine parlamentarische Mehrheit für die Verträge sicher war, aber gleichzeitig auch die Verständigung mit den Partnern nicht scheiterte. Angesichts der Differenzen, die in Spaaks Studienkomitee aufgetreten waren, war das gewiss keine leichte Aufgabe. „Unser Handlungsspielraum war äußerst gering“, formulierte Pineau. ${ }^{10}$

Die Strategie, die die Mollet-Equipe in dieser Situation entwickelte, bestand kurz gesagt darin, zunächst auf Durchsetzung des Euratom-Vertrags zu drängen und dann auf der Grundlage dieses Erfolgs in der französischen Öffentlichkeit einen Meinungsumschwung zugunsten des Gemeinsamen Marktes herbeizuführen. Um noch einmal Pineau zu zitieren, der auf einem Kolloquium in Rom im März 1987 anschaulich über die damaligen Überlegungen berichtete: „Wir mussten eine Art Nebelwand fabrizieren. Euratom war für uns die Nebelwand, hinter der sich der Gemeinsame Markt verborgen hat."11

Diese Strategie ließ sich nicht ohne Abstriche umsetzen. Die deutschen Verhandlungspartner beharrten auf einem Junktim: Wenn schon die ungeliebte Atomgemeinschaft, dann aber auf jeden Fall auch die Wirtschaftsgemeinschaft, so lautete die Verhandlungsdirektive, die das Bundeskabinett beschloss. Die Gegner der Atomgemeinschaft und die Verfechter der Wirtschaftsgemeinschaft in den Reihen der Bundesregierung trafen sich in diesem Junktim. ${ }^{12}$ So musste Pineau auf einer kurzfristig einberufenen Außenministerkonferenz der Sechs am 11./12. Februar 1956 in Brüssel (seiner ersten) der Vorbereitung einer Empfehlung für den Gemeinsamen Markt zustimmen. Gleichzeitig erreichte er aber, dass sein deutscher

7 Vgl. Bruno Riondel: Itinéraire d'un fédéraliste: Maurice Faure, in: Journal of European Integration History 2/ 1997, S. 69-82; Bruno Riondel: Les institutions européennes dans la perspective de M. Faure, in: MarieThérèse Bitsch/Wilfried Loth/Raymond Poidevin (Hrsg.): Institutions européennes et identités europeéennes, Bruxelles 1998, S. 351-379.

8 Christian Pineau: 1956 Suez, Paris 1976, S. 119.

9 Zeugnis auf dem Kolloquium in Rom 25.-28.3.1987, in: Serra: Rilancio, 1989, S. 281-286.

10 Ebenda, S. 283.

11 Ebenda, S. 282-283. Vgl. auch Christian Pineau/Christiane Rimbaud: Le Grand Pari. L'ouverture du traité de Rome, Paris 1991.

12 Zur Kabinettsentscheidung Küsters: Gründung, 1982, S. 256-260; zu den innenpolitischen Widerständen in der Bundesrepublik Wilfried Loth: Deutsche Europa-Konzeptionen in der Gründungsphase der EWG, in: Serra: Rilancio, 1989, S. 585-602, hier S. 591-595. 
Kollege Heinrich von Brentano die Vorbereitung einer Empfehlung für die Atomgemeinschaft akzeptierte. Das war, keine zwei Wochen, nachdem die Regierung Mollet ihr Amt angetreten hatte, der Durchbruch in den Verhandlungen des Spaak-Komitees. Am 20. April wurde die Doppel-Empfehlung zur Schaffung der beiden Gemeinschaften, von Experten im Detail ausgearbeitet, ${ }^{13}$ offiziell von den nationalen Delegationsleitern verabschiedet. ${ }^{14}$

Die Mollet-Equipe versuchte freilich weiterhin, die Atomgemeinschaft vor der Wirtschaftsgemeinschaft zum Abschluss zu bringen, ${ }^{15}$ und Pineau nannte auch gleich drei Bedingungen, die seine Regierung für die Verabschiedung des Vertrags über den Gemeinsamen Markt stellte: Erstens sollten die Überseegebiete in den Gemeinsamen Markt eingeschlossen werden. Zweitens sollten die Sozialleistungen und Steuern in der Gemeinschaft bereits bis zum Ablauf der ersten Integrationsphase weitgehend harmonisiert werden (und zwar nach oben, in Richtung des vergleichsweise hohen französischen Standards). Und drittens sollte der Übergang von der ersten zur zweiten Etappe der Integration nicht automatisch erfolgen; vielmehr sollten die Regierungen die Regelungen für die weiteren Etappen erst nach dem Ablauf der ersten Phase festlegen. Frankreich wollte sich also eine Hintertür offen halten, die Verwirklichung des Gemeinsamen Marktes wenn nötig weiter zu verschieben. ${ }^{16}$

Um sich den nötigen parlamentarischen Rückhalt für das Unternehmen zu verschaffen, organisierte Mollet Anfang Juli 1956 eine Parlamentsdebatte über das Projekt der Atomgemeinschaft. Dabei präzisierte er, dass sich Frankreich die Option auf die Entwicklung eigener Kernwaffen ausdrücklich offen hielt. Den Kernwaffengegnern und den Deutschen, die die französische Sonderstellung nicht auch noch mit ihren Ressourcen fördern wollten, wurde lediglich ein Moratorium von vier oder fünf Jahren zugestanden, in denen es keine französischen Atomwaffentests geben sollte. (In Wirklichkeit war das ein ziemlich theoretisches Moratorium, weil die Entwicklung von testbaren Produkten ohnehin noch in weiter Ferne lag). Außerdem bestand die französische Regierung darauf, dass Frankreich bei der Internationalen Atombehörde selbst und nicht etwa durch die Atomgemeinschaft vertreten sein sollte, und es, anders als der Spaak-Bericht vorsah, keine gemeinsamen Organe von Atomgemeinschaft und Montanunion geben dürfte. Mit diesen Zugeständnissen an die Verfechter einer ,nationalen ' Unabhängigkeitspolitik und einer betont technischen Präsentation des Atomgemeinschafts-Projekts sicherte sich Mollet eine breite Mehrheit für die Fortführung der Verhandlungen über die Atomgemeinschaft (332 gegen 181 Stimmen). ${ }^{17}$

Mit diesem Erfolg im Rücken drängte der französische Verhandlungsleiter Maurice Faure dann in den Vertragsverhandlungen (die am 26. Juni in Val-Duchesse begannen) auf einen raschen Abschluss beider Verträge zu den französischen Bedingungen. ${ }^{18}$ Damit überzog die Mol-

13 Vgl. das Zeugnis von Pierre Uri in Serra: Rilancio, 1989, S. 305-307.

14 Veröffentlicht am 21.4.1956 in allen Gemeinschaftssprachen; ein knapper Auszug aus der deutschen Fassung bei Walter Lipgens (Hrsg.): 45 Jahre Ringen um die europäische Verfassung. Dokumente 1939-1984, Bonn 1986, S. 390-395.

15 Vgl. Pierre Guillen: L'Europe remède à l'impuissance francaise? Le gouvernement Guy Mollet et la négociation des traités de Rome (1955-1957), in: Revue d'histoire diplomatique 102/1988, S. 319-335; Pierre Guillen: La France et la négociation des traités de Rome: L'Euratom, in: Serra: Rilancio, 1989, S. 513-524; sowie das Zeugnis von Maurice Faure, ebenda, S. 286-290.

16 Auszüge aus dem Memorandum, das Pineau seinen Außenministerkollegen vorlegte, bei Robert Marjolin: Le travail d'une vie. Mémoires 1911-1986, Paris 1986, S. 283-286.

17 Vgl. Küsters: Gründung, 1982, S. 294-298, und Peter Weilemann: Die Anfänge der Europäischen Atomgemeinschaft. Zur Gründungsgeschichte von Euratom 1955-1957, Baden-Baden 1983, S. 103-109.

18 Die entsprechende Intervention von Maurice Faure in der Sitzung vom 26.7.1956 (dokumentiert bei Weilemann, Anfänge, 1983, S. 109) zeigt, dass die Regierung Mollet in der Tat nicht bis zur Suez-Krise wartete, ehe sie eine gleichzeitige Unterzeichnung beider Verträge akzeptierte, und bestätigt damit die Kritik von Christian Pineau und Emile Noël (in Serra: Rilancio, 1989, S. 525-527) an der Darstellung von Guillen (ebenda, S. 519). Allerdings sah sie die Verschiebung des Wirtschaftsgemeinschafts-Vertrags weiterhin als Ausweg, wenn die Partner nicht auf ihre Bedingungen eingingen. 
let-Equipe allerdings ihre Position gegenüber den Verhandlungspartnern. Die deutschen Marktwirtschaftler fanden die Forderung nach einer Harmonisierung der Arbeitskosten geradezu absurd; und generell bestand in der Bundesrepublik auch wenig Neigung, Frankreich eine Sonderrolle als militärische Atommacht und Kolonialmacht einzuräumen. Im September gab es eine gewisse Annäherung der Standpunkte, in der Frage der Sozialleistungen und der Einbeziehung der Überseegebiete blieben die Gegensätze jedoch unüberbrückbar. Als sich die deutsche Delegation auf einem erneuten Außenministertreffen am 20./21. Oktober in Paris strikt weigerte, der Reduzierung der wöchentlichen Arbeitszeit von 48 auf 40 Stunden im Laufe der ersten Vertragsphase zuzustimmen, erklärte Pineau die Konferenz für gescheitert. Wieder stand die Zukunft des Projekts in den Sternen; Spaak meinte schon, diesmal sei das Scheitern endgültig. ${ }^{19}$

\section{Der Durchbruch zum Gemeinsamen Markt}

Dass es nicht dabei blieb und die Verhandlungen auch aus dieser Krise wieder herauskamen, ist drei Umständen zu verdanken: Erstens dem Eingreifen Adenauers, der unter allen Umständen einen Erfolg wollte; zweitens einer mutigen Entscheidung von Mollet; und drittens einem in europäischer Hinsicht glücklichen Zufall, der den Meinungsumschwung in der französischen Öffentlichkeit begünstigte.

Zum Ersten: Adenauer wollte nicht nur keinen Rückschlag bei dem Bemühen um weitere Einbindung der Bundesrepublik in die westliche Gemeinschaft zulassen; er glaubte die Wirtschaftsgemeinschaft jetzt auch zustande bringen zu müssen, um die sicherheitspolitische Gemeinschaft zu fördern. Der Radford-Plan zur Reduzierung der amerikanischen Truppenpräsenz in Europa hatte ihm im Sommer 1956 die Gefahr einer amerikanisch-sowjetischen Verständigung auf Kosten der Europäer vor Augen geführt. Dagegen suchte er nun verstärkt die sicherheitspolitische Zusammenarbeit des westlichen Europas; und die sollte nicht durch ein Scheitern der Brüsseler Verhandlungen in Frage gestellt werden. Bei einem Besuch Mollets in Bonn am 29. September hielt Pineau als gemeinsame Überzeugung fest: ,a) Wir sind in Sorge wegen der Entwicklung in Amerika. Wir können uns in Zukunft nicht allein auf die Amerikaner verlassen. b) Die Sechserverhandlungen in Brüssel müssen schnell zu Ende geführt werden. "20 Adenauer fuhr am 6. November nach Paris, um in bilateralen Gesprächen einen Ausweg aus der Verhandlungskrise zu finden, und er ließ sich dabei auch nicht davon stören, dass die Regierung Mollet am Tag zuvor wegen der Suez-Expedition international unter Beschuss geraten war. ${ }^{21}$

Zweitens: Mollet akzeptierte an diesem 6. November eine Kompromissformel, die aus der Verpflichtung zur Anpassung der Sozialleistungen nach oben eine vage Absichtserklärung machte. Die nationale Gesetzgebung sollte wohl darauf hinwirken; spätestens nach sechs Jahren konnte aber mit qualifizierter Mehrheit der Übergang zur zweiten Phase der Integration auch dann beschlossen werden, wenn die Harmonisierung der Lebens- und Arbeitsbedingungen noch nicht erreicht war. Die Kommission konnte in diesem Fall Schutzklauseln für die benachteiligten Industrien erlassen, sie musste es aber nicht. Einfuhrabgaben und Ausfuhrbeihilfen sollten grundsätzlich möglich sein; doch sollten sie allmählich abgebaut werden, und der Gemeinschaft musste darüber jährlich Rechenschaft abgelegt werden. ${ }^{22}$

19 Vgl. Küsters: Gründung, 1982, S. 313-317.

20 Handschriftliches Protokoll Karl Carstens, PAAA.

21 Vgl. hierzu und zum Folgenden Küsters: Gründung, 1982, S. 317-320; Karl Carstens: Das Eingreifen Adenauers in die Europa-Verhandlungen im November 1956, in: Dieter Blumenwitz u.a. (Hrsg.): Konrad Adenauer und seine Zeit. Bd.1: Beiträge von Weg- und Zeitgenossen, Stuttgart 1976, S. 591-602; Pineau/Rimbaud: Le Grand Pari, 1991, S. 223.

22 Vorschlag vom 6.11.1956, PAAA, Büro StS, Bd. 155; vgl. Guillen: L'Europe remède, 1988, S. 331. 
Das bedeutete, nur noch wenig abgemildert, den Sprung der französischen Volkswirtschaft in das kalte Wasser der Konkurrenz des Gemeinsamen Marktes. Mollet wagte ihn, weil ihm die Nationalisierung des Suez-Kanals und die Intervention der Supermächte gegen die britisch-französische Suez-Expedition die Dringlichkeit einer europäischen Atomindustrie vor Augen führten und diese, wie die Verhandlungen gezeigt hatten, nun einmal nicht ohne ein gleichzeitiges und unwiderrufliches Ja zum Gemeinsamen Markt zu haben war. Als guter Taktiker sah er zudem, dass die Demütigung Frankreichs in der Suez-Frage in Verbindung mit dem offenkundigen Abrücken Adenauers von der amerikanischen Führungsmacht Gelegenheit bot, die Hoffnungen auf ein unabhängiges, gegenüber den USA eigenständiges Europa zu reaktivieren. Das war der dritte Faktor, der glückliche Zufall, den Mollet beim Schopf zu packen wusste.

Im Pariser Ministerrat, der vom solidarischen Verhalten des Bundeskanzlers während der Suez-Krise sehr beeindruckt war, setzte Mollet jetzt den Beschluss durch, den Abschluss des Vertrags über die Wirtschaftsgemeinschaft so schnell wie möglich herbeizuführen und sich zu diesem Zweck auch in anderen strittigen Fragen mit der Festlegung ,ziemlich allgemeiner Prinzipien“ zufriedenzugeben, die dann von der „supranationalen Autorität“ der Gemeinschaft präzisiert werden sollten. ${ }^{23}$ Gleichzeitig begann er, das Parlament und die Öffentlichkeit systematisch auf die Notwendigkeit beider Verträge vorzubereiten - und zwar dadurch, dass er sie als Mittel präsentierte, künftig solchen Demütigungen entgehen zu können, wie sie Frankreich durch Nassers Enteignungsmanöver ebenso wie durch die Intervention der Supermächte in der Suez-Krise hatte hinnehmen müssen. Angesichts der quälenden Abhängigkeit vom arabischen Erdöl und der offenkundigen Distanzierung Adenauers von der amerikanischen Führungsmacht erschien ,Europa' zum ersten Mal seit der Zuspitzung der EVG-Krise wieder als Hort der Unabhängigkeit. Dieser Eindruck wurde durch Mollets Propagandaoffensive verstärkt - soweit, dass die öffentliche Meinung nun tatsächlich auch den Gemeinsamen Markt akzeptierte. ${ }^{24}$

Über die Details der Verhandlungen, die jetzt in Erwartung des Erfolgs und entsprechend herzlicher Atmosphäre geführt wurden, ist hier nicht zu berichten. ${ }^{25}$ Wichtig ist in unserem Zusammenhang nur, dass Adenauer Mollet in der Frage der Einbeziehung der Überseegebiete, über die unter dem Druck des französischen Übersee-Ministers Gaston Defferre besonders hartnäckig verhandelt wurde, ${ }^{26}$ schließlich (bei einem letzten Verhandlungstreffen der Regierungschefs am 19./20. Februar 1957) auf halbem Weg entgegenkam, mit einem zeitlich und im Umfang begrenzten Investitionsfonds, und dass Mollet dieses Zugeständnis wieder als ganzen Erfolg zu verkaufen wusste. Um die Formierung einer Oppositionsbewegung schon im Keim zu ersticken, legte Mollet die Verträge, die am 25. März 1957 in Rom unterzeichnet wurden, sogleich der Nationalversammlung vor. Anders als Schuman, der es im Sommer 1952 nicht gewagt hatte, den EVG-Vertrag zur Abstimmung zu stellen, mit dem Ergebnis, dass sich die Aussichten auf eine Ratifizierung von Monat zu Monat verschlechtert hatten.

Den schließlichen Erfolg erlebte Mollet nicht mehr als Regierungschef. Am 21. Mai 1957 wurde er von einer Mehrheit aus Kommunisten, Poujadisten, Konservativen und Radikalen gestürzt, im Wesentlichen, weil diesen ungleichen Verbündeten die dringend notwendige Fi-

23 So die Mitteilung Marjolins in einer Sitzung des interministeriellen Komitees zur Vorbereitung des Gemeinsamen Marktes, zitiert ebenda, S. 332

24 Vgl. Guillen: Euratom, 1993, S. 115-116; Pierre Guillen: Frankreich und der europäische Wiederaufschwung, in: Vierteljahrshefte für Zeitgeschichte 1/1980, S. 1-19, hier S. 16-18.

25 Für einen Überblick vgl. Loth: Deutsche und französische Interessen, 1997, S. 183-185.

26 Vgl. Lafon: Guy Mollet, 2006, S. 496-497. 
nanzreform nicht passte, die die Regierung in Angriff nehmen wollte. Mollet sorgte aber dafür, dass die nächste Regierung unter Maurice Bourgès-Maunoury von den Linksliberalen die bisherige Linie weiter verfolgte. Und Monnet und Maurice Faure arbeiteten im Hintergrund daran, dass die Vorbereitungen zur Ratifizierung der Römischen Verträge zügig fortgesetzt wurden. ${ }^{27}$ Am 9. Juli 1957 stimmte die Nationalversammlung den Verträgen dann tatsächlich zu - mit der überraschend großen Mehrheit von 342 zu 239 Stimmen.

\section{Der zweite Friedensschluss}

Das war, auch wenn es die Öffentlichkeit nicht recht wahrnahm und im öffentlichen Bewusstsein daher bis heute nicht präsent ist, unzweideutig ein Erfolg der Mobilisierungsstrategie, die die Mollet-Equipe Anfang 1956 entwickelt hatte. In gleicher Weise zahlten sich die Zugeständnisse und Gesten aus, zu denen sich Adenauer im Interesse an einer Stabilisierung des deutsch-französischen Gemeinschaftskerns bereit gefunden hatte. Und diese deutsch-französische Achse, die den Kern der Römischen Verträge bildete, wurde dann auch noch einmal sichtbar, als es darum ging, die Verträge in die Praxis umzusetzen. Bei der personellen Ausstattung der Kommission der EWG, der auch für Mollet wichtigeren Gemeinschaft, platzierten die beiden Hauptakteure ihre engsten Mitstreiter in Schlüsselstellungen: Adenauers , rechte Hand“ in der Europapolitik, Walter Hallstein, ${ }^{28}$ wurde erster Präsident der Kommission, und Mollets Vertrauter Emile Noël wurde deren Exekutivsekretär. Dass sich tatsächlich entwickeln konnte, was in den deutsch-französischen Kompromissen von 1956/ 57 angelegt war, ist nicht zuletzt diesen beiden Pionieren europäischer Regierungspraxis zu verdanken. Ihre Zusammenarbeit stellt, historisch gesehen, eine Verstetigung des deutschfranzösischen Gemeinschaftskerns dar.

Dass die Römischen Verträge trotz zweimaliger Gefahr des Scheiterns schließlich doch noch zustande kamen, ist freilich nicht nur der Sensibilität und dem Verhandlungsgeschick des französischen Ministerpräsidenten und des deutschen Bundeskanzlers zuzuschreiben. Die Überzeugungsarbeit der beiden Regierungschefs konnte letztlich nur erfolgreich sein, weil der Vertragskomplex nicht nur in den Details, sondern auch im Grundsätzlichen einen ausgewogenen Kompromiss zwischen französischen und deutschen Interessen enthielt: Die weltmarktorientierte Bundesrepublik band sich an die Sechser-Gemeinschaft mit einem Instrumentarium planerischer und kontraktueller Modernisierungsförderung; dafür wagte Frankreich den Sprung in einen Gemeinsamen Markt, dessen protektionistische Dimension mit Ausnahme der Landwirtschaft immer mehr an Bedeutung zu verlieren drohte.

Ermöglicht wurde dieser Kompromiss durch das gemeinsame Interesse Mollets und Adenauers an Bindung und Selbstbindung der Deutschen und an stärkerer Selbstbehauptung der Europäer gegenüber der amerikanischen Führungsmacht. Insofern dürfte es keine Übertreibung sein, die Römischen Verträge als zweiten Akt des deutsch-französischen Friedensschlusses nach dem Zweiten Weltkrieg zu bezeichnen. Nachdem die Verständigung über die Montanunion einer abermaligen Rivalität um die Hegemonie im Bereich der Schwerindustrie vorgebeugt hatte, verständigte man sich jetzt darauf, als ebenbürtige Partner an der Konstruktion Europas mitzuwirken. ${ }^{29}$

27 Vgl. Jean Monnet: Erinnerungen eines Europäers, München/Wien 1978, S. 513-539; Eric Roussel: Jean Monnet 1888-1979, Paris 1996, S. 715-716.

28 Vgl. Wilfried Loth/Wolfgang Wessels/William Wallace (Hrsg.): Walter Hallstein. Der vergessene Europäer?, Bonn 1985.

29 Vgl. meine Interpretation in Loth: Deutsche und französische Interessen, 1997, S. 187. 